\title{
Realizing the promise of breast cancer vaccines
}

\author{
This article was published in the following Dove Press journal: \\ Vaccine: Development and Therapy \\ 2I August 2012 \\ Number of times this article has been viewed
}

\section{Erica Jackson \\ Hatem Soliman \\ University of South Florida/Moffitt Cancer Center and Research Institute, \\ Tampa, FL, USA}

Correspondence: Hatem Soliman University of South Florida/Moffitt Cancer Center and Research Institute, 12902 Magnolia Drive, Tampa,

FL 336।2, USA

$\mathrm{Tel}+\mathrm{I} 8137454933$

Fax + I 8137457287

Email hatem.soliman@moffitt.org
Abstract: Breast cancer vaccines are being developed to stimulate adaptive antitumor immune responses in patients. These vaccines have the potential to treat breast cancer with minimal side effects and toxicity. However, many obstacles still need to be overcome to fully realize the vaccines' clinical benefit. A review of the literature was conducted to assess the use of vaccines in targeting transformed cells. Four vaccines currently under study were discussed, each summarizing the different vaccine platforms used to introduce target antigen to the patient's immune system. The advantages and disadvantages of each method were discussed, although no one method was found to be superior. Additional issues addressed included overcoming tumor-induced immunosuppression, immune evasion of transformed cells, the use of vaccines in combination therapy, and the challenges of using these vaccines in various clinical settings. Vaccines may be most effective in patients with minimal residual disease, as opposed to using them in the metastatic setting. Also, specific clinical trial design considerations for the use of vaccines in cancer patients, such as time-to-failure end points, were discussed. Understanding these various elements will be important to the translation of breast cancer vaccine therapy into routine clinical practice.

Keywords: breast cancer, vaccine, immunotherapy, immune tolerance, peptide vaccine, dendritic cell vaccine

\section{Importance of the immune system in cancer progression}

The immune system plays a complex and integral role in the evolution of cancer. Hanahan and Weinburg's Hallmarks of Cancer ${ }^{1}$ describes six traits essential for the malignant transformation that takes place as normal cells evolve into cancerous cells. Along with these six original hallmarks, evasion of the immune response has recently been recognized as another capability that must be acquired by tumor cells in order for transformation to occur. ${ }^{1}$

The concept of immunosurveillance embodies the idea that immune cells are constantly circulating through the body, poised to recognize and destroy nascent transformed cells. Detection of cancer cells by the immune system would therefore have to be circumvented in order for a solid tumor to appear. Only those cells that could avoid recognition and elimination would have the capability to progress to the neoplastic state. ${ }^{1}$ It is thought that cancer cells may have the ability to inactivate the cells of the immune system, rendering infiltrating cytotoxic T lymphocytes (CTLs) and natural killer cells inoperative, through the secretion of immunosuppressive factors like transforming growth factor beta. ${ }^{2,3}$ Cancer cells recruit regulatory $\mathrm{T}$ cells and 
myeloid-derived suppressor cells, both an integral part of immunosuppression and CTL inactivation. ${ }^{4,5}$

The immune response can be activated at tumor sites in an attempt to eliminate foreign cells. However, there has recently been evidence demonstrating the tumorpromoting capabilities of the immune response. ${ }^{6-9}$ It was found that tumor-associated macrophages possess the ability to alter the tumor microenvironment through the secretion of various factors. These factors include extracellular matrix enzymes that promote angiogenesis, invasion, metastasis, prevent apoptosis, activate cell growth, and inductive signals that activate the epithelialto-mesenchymal transition. ${ }^{6,9-11}$ There is still much to be understood about the complex role the immune system plays in the development of cancer. The challenge will be how to recruit the immune system in a way that effectively targets malignant cells without unwittingly facilitating their spread. One method of doing this is specifically to activate an adoptive antitumor immune response through the use of effective cancer vaccines.

\section{Using vaccines to target transformed cells}

Based on the premise of immunosurveillance, vaccinations are now being developed to target breast cancer immunologically. Cancer vaccines were developed based on the idea that patients immunized with tumor associated antigens (TAA) could mount effective antitumor immune responses. This precise targeting makes vaccines an attractive method to treat residual disease with little toxicity.

Although research has greatly improved the success of adjuvant treatments, 5-year recurrence rates in breast cancer patients are still around 20\%-30\%. A successful breast cancer vaccine could be used as an adjuvant treatment and reduce this rate of recurrence. Vaccines have shown improved outcomes in patients with lower disease burden, which allows the patient to mount an immune response, before being overtaken by aggressive disease. ${ }^{12,13}$ Vaccines also take longer than cytotoxic treatments to show clinical benefits, as the immune system needs enough time to elicit a strong response. For these reasons, vaccines may be utilized as an effective maintenance therapy in micrometastic disease. However, when used in metastatic patients, vaccines paired with chemotherapy or radiation have shown clinical success. ${ }^{14}$ Due to the need for larger sample sizes and longer follow-up, unselected adjuvant vaccine trials would not be cost effective. However, these studies could prove beneficial in higher-risk groups such as triple-negative patients, who have higher rates of recurrence and shorter recurrence times. ${ }^{15}$ Identifying the most appropriate patients to target with this approach will be critical to the clinical development of cancer vaccines in the adjuvant setting.

Due to the lag time involved in immune system activation, clinical benefit from vaccines may be delayed. Restaging of patients after 6-8 weeks of certain immunotherapies may result in a premature determination of disease progression and removal from study. ${ }^{16}$ For this reason, many studies have found that traditional Response Evaluation Criteria in Solid Tumors (RECIST) guidelines may not be the best way to characterize the clinical benefit in cancer vaccination studies. ${ }^{17-20}$ Evaluating overall survival as an end point may represent a better means to determine vaccine effectiveness, as opposed to time to progression. This would allow vaccination to continue when progression is not rapid and not clinically significant enough to require immediate therapy. ${ }^{20}$

This vaccine approach can also be utilized to target a population of cells known as cancer stem cells (CSC). This approach operates on the idea that a subset of cells exists within the tumor that have the capacity for self-renewal. These CSCs are thought to be responsible for the initiation of disease, resistance to treatment, and occurrence of metastatic disease. ${ }^{21}$ Tumor stem cells are often resistant to radiation and chemotherapy, being left behind to invoke tumor relapse and metastatic disease. ${ }^{21}$ To achieve long-lasting clinical responses, dendritic cell vaccines primed with antigens specific to CSCs may be used to induce the immune response to target and kill this subpopulation of cells..$^{22,23}$

\section{Different vaccine platforms}

This paper will discuss four clinical trials currently studying the clinical efficacy of different breast cancer vaccinations. These vaccinations represent some of the various methods used to introduce target antigens to the patient's immune system. We will discuss the advantages and disadvantages of using peptide-based, viral-based, dendritic cell and plasmid DNA vaccination.

\section{E75 peptide vaccine}

Peptide-based vaccines target tumor-associated antigens, which are differentially overexpressed in tumor cells. Human epithelial receptor 2 (HER2) is a commonly overexpressed antigen in breast cancer tissue and can be targeted by peptide vaccines. ${ }^{24}$ Peptide vaccines are considered human leukocyte antigen (HLA)-restricted because certain HLA subtypes present different pieces of the proteins. This means patients 
must be screened for their HLA type, limiting the amount of patients that can receive the vaccine. In addition, such specific vaccines make it easier for tumors to evade the immune response by expressing lower amounts of the target antigen. For this reason, peptide vaccines targeting multiple epitopes are desirable. ${ }^{25}$ Compared to dendritic cell vaccines where leukapheresis is necessary, peptide vaccines are advantageous because large amounts can be produced with high purity relatively easily. ${ }^{26}$

The E75 vaccine is comprised of a nine-amino acid peptide (HER2 ${ }_{369-377}$ ) that binds to the type I major histocompatibility complex in order to elicit a cytotoxic T-cell response against the HER2 protein. Because peptides are not immunogenic by themselves, the E75 HER2/neu vaccine is administered with granulocyte/macrophage colony-stimulating factor as an adjuvant to stimulate antigen-presenting cells (APCs). Mittendorf et $\mathrm{al}^{27}$ conducted a study with E75 in the adjuvant setting. In $65 \%$ of patients, an immune response was observed with proliferation of E75-specific $\mathrm{CD}^{+} \mathrm{T}$ cells. After 26 months, recurrence rates for vaccinated patients were $8.3 \%$, compared to $14.8 \%$ in the control group. The rate of death in those patients that recurred was $41.7 \%$ in the control group, as opposed to $12.5 \%$ in the vaccinated group.

It is interesting to note that following the peak dose, immunity receded and fell into a plateau phase during the remaining vaccinations. Six months after the completion of vaccinations, only $43 \%$ of patients maintained adequate levels of immunity. ${ }^{27}$ This finding demonstrates the necessity of giving booster shots to those patients at higher risk of recurrence. It is also likely that a prolonged immune response will require the activation of both CTLs and helper T lymphocytes (HTLs), which can be accomplished by fusing peptides that contain HTL- and CTL-activating portions. $^{28-31}$

Previous data indicate that vaccination with an E75 peptide can generate epitope spreading. ${ }^{32}$ Epitope spreading to GP2, a subdomain of the HER2/neu protein was observed in all node-positive patients and $85 \%$ of node-negative patients. ${ }^{33}$ Because single-peptide vaccines do not exhibit antigen diversity, epitope spreading may partially overcome the need for multiple-antigen stimulation. However, a more effective strategy may still require a vaccine against multiple epitopes or an E75 vaccine in combination with trastuzumab in HER2-positive patients. ${ }^{26,34}$ Currently, E75 is undergoing evaluation in a multinational phase III registration (PRESENT) study for node-positive, HLA A2/A3 patients with HER2 $1-2^{+}$by immunohistochemistry.

\section{MUC-I/CEA/TRICOM viral vector vaccine}

PANVAC is a recombinant poxviral vaccine that expresses carcinoembryonic antigen (CEA) and mucin-1 (MUC-1), in addition to three costimulatory molecules named TRICOM. TRICOM consists of three T-cell costimulatory molecules, including B7-1, intercellular adhesion molecule 1, and lymphocyte function-associated antigen 3 . When expressed in APCs, TRICOM has been shown to elicit a stronger immune response, along with a greater number of $\mathrm{T}$ cells specific for the TAA. ${ }^{35-37}$ Viral vaccinations are able to elicit an immune response by inducing antigen presentation in the APCs that take up the virus. Using the poxviral vaccine allows the insertion of multiple different genes that can then be expressed in host cells following vaccination. ${ }^{38}$ One downfall of viral vaccinations is that neutralizing antiviral antibodies are produced following the initial vaccination, rendering subsequent vaccinations ineffective. Therefore, further booster vaccination must utilize a fowlpox vaccine.$^{39}$ In a study by Mohebtash et al, ${ }^{16}$ twelve metastatic breast cancer patients had a median time to progression of 2.5 months following vaccination with PANVAC. Five of the twelve breast patients had stable disease or tumor regression for 4 months or more. These five responders had a mean of 1.8 chemotherapies prior to vaccination and lower tumor-marker levels. Conversely, nonresponders had a mean of four previous chemotherapy treatments and much higher mean serum CEA levels. This study showed that patients with a smaller tumor burden and a lower number of previous chemotherapy treatments responded better to vaccine therapy. Patients also showed increased response to subsequent therapies, such as chemotherapy or radiation. Following vaccination, these cytotoxic therapies may reduce the immunosuppressor cells present at the tumor site, allowing a stronger T-cell response to occur. ${ }^{17,40,41}$

\section{Lapuleucel-T autologous dendritic cell vaccine}

APCs are responsible for the uptake, processing, and presentation of antigens to T cells. Leukapheresed APCs can be activated with TAAs ex vivo and reintroduced into patients to present these antigens to effector $\mathrm{T}$ cells, thereby activating the immune system. ${ }^{42}$ Because this method uses autologous cells, this type of vaccine is compatible with any patient. However, one disadvantage of this approach is the need for leukapheresis in order to generate the primed cells. This presents issues such as inconsistent product due to varying yields of activated APCs, the need for leukapheresis facilities, 
and the possible need for indwelling catheters. ${ }^{43}$ However, this approach has produced the only FDA-approved cancer vaccine therapy to date Provenge ${ }^{\circledR}$ (sipuleucel-T) (Dendreon Corp, Seattle, WA) for hormone-refractory metastatic prostate cancer. A phase 1 trial studying the lapuleucel-T vaccination introduced autologous APCs loaded with multiple HER2/neu sequences into metastatic breast cancer patients. ${ }^{44}$ As opposed to peptide and dendritic cell-based approaches, this method can induce immune responses to multiple HER2/neu antigens with little to no toxicities reported. ${ }^{43}$ The results from the lapuleucel study showed the median time to disease progression was 12.8 weeks, with two breast cancer patients showing stable disease after 48 weeks. One patient was even treated with lapuleucel-T 1 year later, and had stable disease at week 72. It is possible that this vaccine could be utilized in high risk individuals with minimal residual HER2 expressing disease to prevent recurrence.

\section{Cytochrome P450 IBI (CYPIBI) plasmid DNA vaccine}

This plasmid DNA vaccine was designed to stimulate an immune response against CYP1B1 epitopes commonly expressed on the surface of transformed cells. Seventeen patients with advanced stage disease underwent vaccination to determine safety and immunogenicity of this approach. ${ }^{40}$ The vaccine contains plasmid DNA encoding an inactivated form of CYP1B1. While six of the 17 patients developed CYP1B1-specific immunity, it is interesting to note that five of the patients that developed immunity exhibited a significant response to their next salvage therapy. Of the eleven that did not develop immunity, three died before receiving salvage therapy, seven progressed after salvage therapy, and one had complete remission. There is increasing evidence that once a vaccine activates the immune system, patients have a better response to subsequent therapies. ${ }^{40} \mathrm{This}$ group hypothesized that immunity to CYP1B1 somehow primes for the next-line treatment through the alteration of the tumor microenvironment and tumor-cell resistance. This could be attributed to numerous mechanisms, including alteration of tumor phenotype, antigen cascade, and reduction in immune suppressor cells. ${ }^{45-47}$

This group looked to determine reasons why some patients developed immunity and some did not. The only difference that seemed predictive of the acquisition of immunity was the number of prior treatments received. It was thought that patients with a greater number of previous treatments would not be as capable of developing an immune response. These patients did in fact have a decreased response to phytohemagglutinin; however, they were able to elicit a normal T-cell response against a pool of CEF-specific peptides (pooled peptides from cytomegalovirus, EpsteinBarr virus, and influenza virus). In addition, the number of vaccinations had no effect on immunity, as all six patients developed immunity between vaccinations two and six, with no increase in immunity during further vaccination. One explanation could be that aggressive disease inhibits the patient's ability to generate immunity to the TAA, and responders inherently had a slower-growing cancer. It was also theorized that the tumor burden might be selective for the level of immune response generated, due to regulatory $\mathrm{T}$ cells and immunosuppressive cytokines. ${ }^{48}$

\section{Tumor-mediated immunosuppression}

One of the obstacles that must be overcome to develop a more effective breast cancer vaccine is the concept of immune escape. Tumor cells can often evolve mechanisms to escape recognition by the immune system. This occurs when an outgrowth of new tumor cells no longer expresses the target antigen. ${ }^{49}$ Using vaccines that target multiple tumorassociated antigens may provide a means to avoid immune escape. There has also been some interest in polyvalent vaccines that stimulate both cell-mediated and humoral-mediated immunity. ${ }^{28-31}$

Inhibitory signals can also be utilized by the tumor to evade immune recognition. This is especially important in the use of vaccines in the locally advanced or metastatic settings. Blockade of these inhibitory signals can be used to augment vaccinations and create a more robust immune response. Cytotoxic T-lymphocyte antigen 4 (CTLA-4) inhibition of CTLs can be blocked using the CTLA-4 antibodies, ipilimumab, and tremelimumab. ${ }^{50-52}$ Many breast cancer patients often express the inhibitory signal B7-H1 on APCs, which can bind programmed cell death 1 (PD-1) of lymphocytes, rendering them anergic. Anti-PD-1 antibodies would allow lymphocytes to destroy tumor cells more effectively without being inactivated by the B7-H1 inhibitory signal. $^{53,54}$

Although cells are primed with the TAA and ready to attack malignant cells, the hostile tumor microenvironment can secrete immunosuppressors, inducing anergy in the infiltrating cells. ${ }^{55,56}$ Indoleamine 2,3 dioxygenase (IDO) is a tryptophan-catabolizing enzyme that inhibits the immune response and can be exploited by tumor cells to help prevent rejection by the immune system. When IDO is overexpressed in the tumor microenvironment, it causes production of metabolites that are toxic to infiltrating $\mathrm{T}$ cells, 
rendering them inactive. ${ }^{57-59}$ IDO also induces proliferation of regulatory $\mathrm{T}$ cells, thereby suppressing the response to tumor antigens. 1-Methyl tryptophan is a competitive inhibitor of IDO, and in previous studies has been shown to increase antitumor T cells, thereby slowing tumor growth. ${ }^{60}$ Tumors can also suppress the immune response by secreting cytokines like transforming growth factor beta that can inhibit infiltrating effector $\mathrm{T}$ cells. It has been shown that using an antibody against these inhibitory cytokines could potentially block their immunosuppressive function. ${ }^{61}$

\section{Combination therapy}

One way to elicit a stronger immune response and possibly derive clinical benefit would be to combine vaccines with other cancer treatments, such as chemotherapy, monoclonal antibodies, or radiation. One study showed patients with improved clinical outcomes when receiving the PANVAC vaccine plus the chemotherapy agent docetaxel, as opposed to PANVAC or docetaxel alone. ${ }^{62}$ It is thought that vaccines alter the tumor microenvironment, making cells more susceptible to the cytotoxic effects of chemotherapy and radiation. ${ }^{45-48}$

In a recent study, "vaccine sensitizing" was studied by treating breast cancer cells with trastuzumab before administering the E75 HER2/neu peptide vaccine. ${ }^{34}$ An increased CTL response against the E75 peptide was observed, possibly due to the increased processing of the HER2/neu antigen. This would provide a greater number of peptides available to be presented by the APC, and potentially a greater number of tumor cells that are recognized and eliminated..$^{34}$

\section{Neoadjuvant versus metastatic setting}

Vaccines have also been proposed as a means to elicit antitumor immunity in an adjuvant setting with patients who have minimal residual disease. ${ }^{63}$ Although most vaccine trials have enlisted metastatic patients with a much larger tumor burden, using vaccines in the adjuvant setting could reduce the time to recurrence. However, for vaccinations to be effective as an adjuvant therapy, long-term memory must be elicited without causing autoimmunity. ${ }^{64}$ Another factor that would need to be addressed is the outgrowth of tumor cells lacking antigen expression, thereby evading the immune response. ${ }^{49}$

\section{Prevention of breast cancer using vaccines}

Jaini et a ${ }^{65}$ studied the effects of the dendritic cell vaccine alpha-lactalbumin, a unique antigen expressed only during lactation. It was hypothesized that vaccination with alpha-lactalbumin in women past the age of childbearing years would be a safe and effective preventive therapy for high-risk breast cancer patients. In the breast tissue of nonlactating mice, immunization with alpha-lactalbumin caused $\mathrm{T}$ cells to migrate through the tissue in a classic surveillance manner. In lactating tissue, inflammation due to the T-cell response actually caused breast failure. Alpha-lactalbumin therefore would likely need to be used for prevention in high risk women who no longer wish to breastfeed.

\section{Conclusion}

Breast cancer vaccines provide a means to elicit an immune response to antigens specifically expressed by tumor cells. There are different ways of introducing these antigens, including peptide-based, viral-based, dendritic cell, and plasmid DNA vaccines. Each method has distinct advantages and disadvantages. Because the metastatic setting may not allow vaccines the proper amount of time to induce an immune response, these vaccines, when used alone, may be more effective in the minimal residual disease state. Assessing the clinical benefit of these agents will require the use of different end points (likely time to event/failure) rather than objective responses per RECIST. While there have been some advances in the development of breast cancer vaccines, much work remains to be done in order to fully maximize their potential. Identifying the individuals who would most benefit from vaccination, discovering which vaccines would work best in a given setting, how to integrate them with other treatment modalities, and overcoming tumor-related immunosuppression are all important issues that must be addressed. The ultimate goal is to one day be able to use vaccines specifically to stimulate a patient's immune system, while minimizing toxicity and side effects.

\section{Disclosure}

The authors report no conflicts of interest in this work.

\section{References}

1. Hanahan D, Weinberg RA. Hallmarks of cancer: the next generation. Cell. 2011;144(5):646-674.

2. Shields JD, Kourtis IC, Tomei AA, Roberts JM, Swartz MA. Induction of lymphoidlike stroma and immune escape by tumors that express the chemokine CCL21. Science. 2010;328(5979):749-752.

3. Yang L, Pang Y, Moses HL. TGF-beta and immune cells: an important regulatory axis in the tumor microenvironment and progression. Trends Immunol. 2010;31(6):220-227.

4. Mougiakakos D, Choudhury A, Lladser A, Kiessling R, Johansson CC. Regulatory T cells in cancer. Adv Cancer Res. 2010;107:57-117.

5. Ostrand-Rosenberg S, Sinha P. Myeloid-derived suppressor cells: linking inflammation and cancer. J Immunol. 2009;182(8):4499-4506. 
6. DeNardo DG, Andreu P, Coussens LM. Interactions between lymphocytes and myeloid cells regulate pro- versus anti-tumor immunity. Cancer Metastasis Rev. 2010;29(2):309-316.

7. Dvorak HF. Tumors: wounds that do not heal. Similarities between tumor stroma generation and wound healing. $N$ Engl J Med. 1986; 315(26):1650-1659.

8. Colotta F, Allavena P, Sica A, Garlanda C, Mantovani A. Cancer-related inflammation, the seventh hallmark of cancer: links to genetic instability. Carcinogenesis. 2009;30(7):1073-1081.

9. Grivennikov SI, Greten FR, Karin M. Immunity, inflammation, and cancer. Cell. 2010;140(6):883-899.

10. Qian BZ, Pollard JW. Macrophage diversity enhances tumor progression and metastasis. Cell. 2010;141(1):39-51.

11. Karnoub AE, Dash AB, Vo AP, et al. Mesenchymal stem cells within tumour stroma promote breast cancer metastasis. Nature. 2007; 449(7162):557-563.

12. Wright SE, Rewers-Felkins KA, Quinlin IS, et al. Tumor burden influences cytotoxic $\mathrm{T}$ cell development in metastatic breast cancer patients - a phase I/II study. Immunol Invest. 2009;38(8):820-838.

13. Wolchok JD, Hoos A, O’Day S, et al. Guidelines for the evaluation of immune therapy activity in solid tumors: immune-related response criteria. Clin Cancer Res. 2009;15(23):7412-7420.

14. Antonia SJ, Mirza N, Fricke I, et al. Combination of p53 cancer vaccine with chemotherapy in patients with extensive stage small cell lung cancer. Clin Cancer Res. 2006;12(3 Pt 1):878-887.

15. Dent R, Trudeau M, Pritchard KI, et al. Triple-negative breast cancer: clinical features and patterns of recurrence. Clin Cancer Res. 2007; 13(15 Pt 1):4429-4434.

16. Mohebtash M, Tsang KY, Madan RA, et al. A pilot study of MUC-1/ CEA/TRICOM poxviral-based vaccine in patients with metastatic breast and ovarian cancer. Clin Cancer Res. 2011;17(22):7164-7173.

17. Schlom J, Arlen PM, Gulley JL. Cancer vaccines: moving beyond current paradigms. Clin Cancer Res. 2007;13(13):3776-3782.

18. Finke LH, Wentworth K, Blumenstein B, Rudolph NS, Levitsky H, Hoos A. Lessons from randomized phase III studies with active cancer immunotherapies - outcomes from the 2006 meeting of the Cancer Vaccine Consortium (CVC). Vaccine. 2007;25 Suppl 2: B97-B109.

19. Kantoff PW, Schuetz TJ, Blumenstein BA, et al. Overall survival analysis of a phase II randomized controlled trial of a Poxviral-based PSA-targeted immunotherapy in metastatic castration-resistant prostate cancer. J Clin Oncol. 2010;28(7):1099-1105.

20. Hoos A, Parmiani G, Hege K, et al. A clinical development paradigm for cancer vaccines and related biologics. J Immunother. 2007;30(1): $1-15$.

21. Morrison BJ, Schmidt CW, Lakhani SR, Reynolds BA, Lopez JA. Breast cancer stem cells: implications for therapy of breast cancer. Breast Cancer Res. 2008;10(4):210.

22. Dhodapkar MV, Dhodapkar KM. Vaccines targeting cancer stem cells: are they within reach? Cancer J. 2011;17(5):397-402.

23. Ning N, Pan Q, Zheng F, et al. Cancer stem cell vaccination confers significant antitumor immunity. Cancer Res. 2012;72(7): 1853-1864.

24. Vlad AM, Finn OJ. Glycoprotein tumor antigens for immunotherapy of breast cancer. Breast Dis. 2004;20:73-79.

25. Buteau C, Markovic SN, Celis E. Challenges in the development of effective peptide vaccines for cancer. Mayo Clin Proc. 2002;77(4): 339-349.

26. Mittendorf EA, Peoples GE, Singletary SE. Breast cancer vaccines: promise for the future or pipe dream? Cancer. 2007;110(8):1677-1686.

27. Mittendorf EA, Holmes JP, Ponniah S, Peoples GE. The E75 HER2/ neu peptide vaccine. Cancer Immunol Immunother. 2008;57(10): 1511-1521.

28. Kawashima I, Hudson SJ, Tsai V, et al. The multi-epitope approach for immunotherapy for cancer: identification of several CTL epitopes from various tumor-associated antigens expressed on solid epithelial tumors. Hum Immunol. 1998;59(1):1-14.
29. Kobayashi H, Nagato T, Oikawa K, et al. Recognition of prostate and breast tumor cells by helper $\mathrm{T}$ lymphocytes specific for a prostate and breast tumor-associated antigen, TARP. Clin Cancer Res. 2005;11(10): 3869-3878.

30. Lu J, Higashimoto Y, Appella E, Celis E. Multiepitope Trojan antigen peptide vaccines for the induction of antitumor CTL and Th immune responses. J Immunol. 2004;172(7):4575-4582.

31. Kobayashi H, Celis E. Peptide epitope identification for tumor-reactive CD4 T cells. Curr Opin Immunol. 2008;20(2):221-227.

32. Disis ML, Gooley TA, Rinn K, et al. Generation of T-cell immunity to the HER-2/neu protein after active immunization with HER-2/neu peptide-based vaccines. J Clin Oncol. 2002;20(11):2624-2632.

33. Mittendorf EA, Gurney JM, StorrerCE, ShriverCD, Ponniah S, Peoples GE. Vaccination with a HER2/neu peptide induces intra- and inter-antigenic epitope spreading in patients with early stage breast cancer. Surgery. 2006;139(3):407-418.

34. Mittendorf EA, Storrer CE, Shriver CD, Ponniah S, Peoples GE. Investigating the combination of trastuzumab and HER2/neu peptide vaccines for the treatment of breast cancer. Ann Surg Oncol. 2006;13(8): 1085-1098.

35. Hodge JW, Sabzevari H, Yafal AG, Gritz L, Lorenz MG, Schlom J. A triad of costimulatory molecules synergize to amplify T-cell activation. Cancer Res. 1999;59(22):5800-5807.

36. Grosenbach DW, Barrientos JC, Schlom J, Hodge JW. Synergy of vaccine strategies to amplify antigen-specific immune responses and antitumor effects. Cancer Res. 2001;61(11):4497-4505.

37. Hodge JW, Chakraborty M, Kudo-Saito C, Garnett CT, Schlom J. Multiple costimulatory modalities enhance CTL avidity. J Immunol. 2005;174(10):5994-6004.

38. Essajee S, Kaufman HL. Poxvirus vaccines for cancer and HIV therapy. Expert Opin Biol Ther. 2004;4(4):575-588.

39. Marshall JL, Hoyer RJ, Toomey MA, et al. Phase I study in advanced cancer patients of a diversified prime-and-boost vaccination protocol using recombinant vaccinia virus and recombinant nonreplicating avipox virus to elicit anti-carcinoembryonic antigen immune responses. J Clin Oncol. 2000;18(23):3964-3973.

40. Gribben JG, Ryan DP, Boyajian R, et al. Unexpected association between induction of immunity to the universal tumor antigen CYP1B1 and response to next therapy. Clin Cancer Res. 2005;11(12):4430-4436.

41. Arlen PM, Gulley JL, Parker C, et al. A randomized phase II study of concurrent docetaxel plus vaccine versus vaccine alone in metastatic androgen-independent prostate cancer. Clin Cancer Res. 2006;12(4): 1260-1269.

42. Park JW, Melisko ME, Esserman LJ, Jones LA, Wollan JB, Sims R. Treatment with autologous antigen-presenting cells activated with the HER-2 based antigen Lapuleucel-T: results of a phase I study in immunologic and clinical activity in HER-2 overexpressing breast cancer. J Clin Oncol. 2007;25(24):3680-3687.

43. Soliman H. Developing an effective breast cancer vaccine. Cancer Control. 2010;17(3):183-190.

44. Peethambaram PP, Melisko ME, Rinn KJ, et al. A phase I trial of immunotherapy with lapuleucel-T (APC8024) in patients with refractory metastatic tumors that express HER-2/neu. Clin Cancer Res. 2009;15(18):5937-5944.

45. Kawaida H, Kono K, Takahashi A, et al. Distribution of CD4+ CD25 high regulatory T-cells in tumor-draining lymph nodes in patients with gastric cancer. J Surg Res. 2005;124(1):151-157.

46. Garnett CT, Palena C, Chakraborty M, Tsang KY, Schlom J, Hodge JW. Sublethal irradiation of human tumor cells modulates phenotype resulting in enhanced killing by cytotoxic T lymphocytes. Cancer Res. 2004;64(21):7985-7994.

47. Chakraborty M, Abrams SI, Camphausen K, et al. Irradiation of tumor cells up-regulates Fas and enhances CTL lytic activity and CTL adoptive immunotherapy. J Immunol. 2003;170(12):6338-6347.

48. Curiel TJ, Coukos G, Zou L, et al. Specific recruitment of regulatory $\mathrm{T}$ cells in ovarian carcinoma fosters immune privilege and predicts reduced survival. Nat Med. 2004;10(9):942-949. 
49. Jäger E, Ringhoffer M, Karbach J, Arand M, Oesch F, Knuth A. Inverse relationship of melanocyte differentiation antigen expression in melanoma tissues and CD8+ cytotoxic-T-cell responses: evidence for immunoselection of antigen-loss variants in vivo. Int $J$ Cancer. 1996;66(4):470-476.

50. Leach DR, Krummel MF, Allison JP. Enhancement of antitumor immunity by CTLA-4 blockade. Science. 1996;271(5256):1734-1736.

51. Pilones KA, Kawashima N, Yang AM, Babb JS, Formenti SC, Demaria S. Invariant natural killer $\mathrm{T}$ cells regulate breast cancer response to radiation and CTLA-4 blockade. Clin Cancer Res. 2009;15(2): 597-606.

52. Dewan MZ, Galloway AE, Kawashima N, et al. Fractionated but not single-dose radiotherapy induces an immune-mediated abscopal effect when combined with anti-CTLA-4 antibody. Clin Cancer Res. 2009;15(17):5379-5388.

53. Ghebeh H, Barhoush E, Tulbah A, Elkum N, Al-Tweigeri T, Dermime S. FOXP3+ Tregs and B7-H1+/PD-1+ T lymphocytes co-infiltrate the tumor tissues of high-risk breast cancer patients: implication for immunotherapy. BMC Cancer. 2008;8:57.

54. Ghebeh H, Mohammed S, Al-Omair A, et al. The B7-H1 (PD-L1) T lymphocyte-inhibitory molecule is expressed in breast cancer patients with infiltrating ductal carcinoma: correlation with important high-risk prognostic factors. Neoplasia. 2006;8(3):190-198.

55. Gajewski TF. Identifying and overcoming immune resistance mechanisms in the melanoma tumor microenvironment. Clin Cancer Res. 2006;12(7 Pt 2):2326s-2330s.

56. Mellor AL, Munn DH. Creating immune privilege: active local suppression that benefits friends, but protects foes. Nat Rev Immunol. 2008;8(1):74-80.
57. Fallarino F, Grohmann U, Vacca C, et al. T cell apoptosis by tryptophan catabolism. Cell Death Differ. 2002;9(10):1069-1077.

58. Friberg M, Jennings R, Alsarraj M, et al. Indoleamine 2,3-dioxygenase contributes to tumor cell evasion of $\mathrm{T}$ cell-mediated rejection. Int $J$ Cancer. 2002;101(2):151-155.

59. Frumento G, Rotondo R, Tonetti M, Damonte G, Benatti U, Ferrara GB. Tryptophan-derived catabolites are responsible for inhibition of $\mathrm{T}$ and natural killer cell proliferation induced by indoleamine 2,3-dioxygenase. J Exp Med. 2002;196(4):459-468.

60. Muller AJ, DuHadaway JB, Donover PS, Sutanto-Ward E, Prendergast GC. Inhibition of indoleamine 2,3-dioxygenase, an immunoregulatory target of the cancer suppression gene Bin1, potentiates cancer chemotherapy. Nat Med. 2005;11(3):312-319.

61. Wojtowicz-Praga S, Verma UN, Wakefield L, Esteban JM, Hartmann D, Mazumder A. Modulation of B16 melanoma growth and metastasis by anti-transforming growth factor beta antibody and interleukin-2. J Immunother Emphasis Tumor Immunol. 1996;19(3):169-175.

62. Arlen PM, Pazdur M, Skarupa L, Rauckhorst M, Gulley JL. A randomized phase II study of docetaxel alone or in combination with PANVAC-V (vaccinia) and PANVAC-F (fowlpox) in patients with metastatic breast cancer (NCI 05-C-0229). Clin Breast Cancer. 2006;7(2):176-179.

63. Finn OJ. Cancer vaccines: between the idea and the reality. Nat Rev Immunol. 2003;3(8):630-641.

64. Lollini PL, Cavallo F, Nanni P, Forni G. Vaccines for tumour prevention. Nat Rev Cancer. 2006;6(3):204-216.

65. Jaini R, Kesaraju P, Johnson JM, Altuntas CZ, Jane-Wit D, Tuohy VK. An autoimmune-mediated strategy for prophylactic breast cancer vaccination. Nat Med. 2010;16(7):799-803

\section{Publish your work in this journal}

Vaccine: Development and Therapy is an international, peer-reviewed, open access journal that spans the spectrum of vaccine design and development through to clinical applications. The journal is characterized by the rapid reporting of application notes, reviews, original research and clinical studies in all therapeutic areas. Clinical outcomes, patient safety, and programs for the development and effective, safe, and sustained use of vaccines will be a feature of the journal. The manuscript management system is completely online and includes a very quick and fair peer-review system. Visit http://www.dovepress.com/testimonials.php to read real quotes from published authors. 